\author{
Asian Journal of \\ Medical and Biological Research \\ ISSN 2411-4472 (Print) 2412-5571 (Online) \\ www.ebupress.com/journal/ajmbr
}

\title{
Article \\ Effects of yoga on flexibility and balance: a quasi-experimental study
}

\author{
Shah Noman Md. Iftekher ${ }^{1}$, Md. Bakhtiar ${ }^{2}$ and Kh. Shafiur Rahaman ${ }^{3 *}$ \\ ${ }^{1}$ Post Graduate Diploma in Exercise Physiology student, Department of Exercise Physiology, Institute of Sports \\ Science, Bangladesh Krira Shikkha Prathisthan (BKSP), Dhaka, Bangladesh \\ ${ }^{2}$ Senior Research Officer, Department of Exercise Physiology, Institute of Sports Science, Bangladesh Krira \\ Shikkha Prathisthan (BKSP), Dhaka, Bangladesh \\ ${ }^{3}$ GIBACHT fellow, German Partnership Program for Excellence in Biological and Health Security, Germany
}

*Corresponding author: Kh. Shafiur Rahaman, BSL Residential Complex, Flat \# Bakul 804, Mirpur-13, Dhaka1216, Bangladesh. Phone: +880 1670215 932; Email: rajib_pt@yahoo.com

Received: 07 June 2017/Accepted: 25 June 2017/ Published: 29 June 2017

\begin{abstract}
Despite having number of health benefits, yoga also has a proven role in enhancing performance of athletes boosting specific components of fitness. Our aim was to study the effect of yoga on flexibility and balance among shooting trainee athletes at BKSP, Bangladesh. A quasi-experimental study was conducted among shooting trainee athletes. In total 20 athletes took part in this study. In each group we had 10 participants (10 in yoga group and other 10 in non-yoga group). Regular yoga session have been conducted early morning biweekly over a period of six weeks. All the participants were allowed to take part in regular training session, while only yoga group took part in additional yoga session. Measurements of flexibility and balance including Sit and Reach (SR) test and Stork Stand (SR) test were taken immediately before and after the yoga training period. Independent t-test and paired t-test were used to determine the significant effect of yoga within and between the groups before and after yoga training. Sixty percent of our participants were male. Participant's age were between 12-17 years. All of them had normal level of BMI. Significant improvement were observed in the yoga group for flexibility $(\mathrm{SR}, \mathrm{P}=0.017)$ and balance $(\mathrm{SS}, \mathrm{P}=0.004)$ during within group comparison. No significant improvement were seen for flexibility and balance in the non-yoga group. Between group comparison (Yoga and Non-yoga) also shows significant enhancement in both flexibility (SR, $\mathrm{P}=0.018$ ) and balance (SS, $\mathrm{P}=0.021$ ). Our findings helped us to conclude that regular yoga training may improve the balance and flexibility of shooting athletes even within short period of time (6 weeks), can also improve the athletic performances that demands high flexibility and balance.
\end{abstract}

Keywords: yoga; flexibility; balance; athletes; shooting game

\footnotetext{
1. Introduction

Regular practice of yoga has numerous health benefits (Wolff et al., 2013; Pal et al., 2011; McDermott et al., 2014; Parikh et al., 2014). Yoga also brings positive changes in physical performance and well-being if practiced regularly (Akhtar et al., 2013; Ross and Thomas, 2010) by improving flexibility and balance (Boehde et al., 2005) as well as cardiovascular functions (Bera and Rajapurkar, 1993). Moreover, yoga may have direct link to improve the common elements of athletic performance (Harrelson and Swann, 2003). It is also evident that yoga can be an important element of training program along with the other regular traditional exercise or even may replace those (Broad, 2012). Regular yoga practice results in enhanced flexibility very rapidly as this process involves gentle stretching of muscle, connective tissues around bones and joints (Woodyard, 2011). Yoga also has profound effect on balance, muscular strength, endurance and coordination because of its highly structural activity and involvement (Carrico, 1997).
} 
Yoga differs from other typical forms of exercise training as it requires multi-structural involvement that gives a difficult task to the body in various ways (Gulati and Sharma, 2011; Kaminoff and Matthews, 2007). Proper positioning trough yoga enhances movement abilities and reduces movement limitation, thus improves body functioning among athletes. It also helps to maintain continuous and stable breathing through a series of Asanas (static posture) involving required muscle groups under tension. Interacting breathing mechanism to the tensed musculoskeletal system brings comprehensive changes to the whole body while performing those Asanas (Coulter, 2010).

Traditional exercise emphasizes on improving specific fitness for a given sports achievement (Bryant and Green, 2006). Though specific components of fitness increases, it is difficult to use these in attaining optimal athletic performance (Aaberg, 2002). On the other hand, regular yoga practice improves many specific components of fitness (e.g. increase alignment, increase range of motion, and enhance muscle fibers recruitment) by increasing flexibility and reducing muscle tension thus allows new movements to take place and help joints to move freely (Clark and Powers, 2012). Thus sport skill improves.

In shooting game, balance and flexibility is important for athletes to hold their gun for longer period and without bouncing it. According to the evidence above, yoga helps to strengthen and refine connective muscle tissue and some of those really small muscle that are responsible for balance and stillness. A good practice of yoga could be of great help for shooting athletes in this regard.

Therefore, the main intention of this paper was to investigate the effect of yoga on specific components of fitness particularly flexibility and balance related to athletic performance. Thus, through an improvement of specific fitness elements, the core ability for athletic performance should increase. To apply our results to competitive situations, we performed our experiment on trainee athletes who were participating in regular training at shooting department of Bangladesh Krira Shikkha Prathisthan (BKSP), Bangladesh for their athletic events.

\section{Materials and Methods}

A quasi-experimental study was carried out for a period of 7 weeks (Measurement days and yoga training session) to assess the impact of yoga on specific aspects of athletic fitness among the suitable sportsperson participating in regular shooting training at BKSP. The study measurements were taken in the Department of Exercise Physiology at Institute of Sports Science, BKSP and the yoga training session was conducted in the shooting complex of BKSP. The study duration was from April, 2017 to May, 2017. Yoga training were given over a period of 6 weeks at a frequency of twice per week. We have selected 20 participants according to their availability and suitability guided by the coaches allowing the researchers to collect the data on their athletes. Participants $(n=20)$ were trainee athletes in shooting department both male and female. The subjects were divided among yoga group $(n=10)$ and non-yoga group $(n=10)$.The participants had no previous experience with yoga and were free from injury. History of any existing medical condition were also asked before including them in this study. Students who passed at least one year training at BKSP were included. New students were excluded from the study as without particular sports training it can bias our study hypothesis.

\subsection{Procedures}

The yoga group and the non-yoga group was comprised of shooting trainee players. During the period of yoga sessions for 6 weeks, members of both group have also participated the regularly pre-scheduled sport specific training. Common training included static stretching exercises, weight and endurance training, and running for both groups. In addition to their regular training, the yoga subjects took part in yoga sessions in early morning (Monday and Thursday) each week before any other physical activity. Sessions were conducted by professional yoga expert. The yoga expert demonstrated variety of yoga poses (Asanas) and the participants then followed and imitated those poses. Each session took place for an hour. Measures of flexibility and balance were taken immediately before and shortly after the 6-week yoga sessions.

Assessments for each group were completed separately. One day before the initiation of first yoga session, the measurements were taken with the same testing protocol from both yoga and non-yoga group members. Similarly, at the end of 6 weeks yoga training session, one day after, the testing protocol was repeated with the yoga group and non-yoga group athletes respectively. Without any warm up session, the following assessment protocol was completed: (1) Sit-reach (SR) test and (2) Stork stand (SS) test. The measures of flexibility were determined by an SR test (Baechle, 2008), while a test of balance was conducted with a stork stand (SS) test (Coulson and Archer, 2011). We have reported the best attempts out of three. 


\subsection{Statistical analysis}

Standard descriptive statistics (mean \pm standard deviation) were determined for directly measured and derived variables. Before-after comparison of means were calculated for all measured values. Paired t-test were used for the comparison of various characteristic variables between two groups. Data were analyzed using SPSS (Statistical Package for Social Science) version 22.0. A 5\% level of probability was used to indicate statistical significance.

\section{Results}

A total number of 20 participants (10 from each group, i.e. yoga and non-yoga group) were included in this study. Participants were both male and female trainee athletes of shooting game at BKSP. The Mean age of the participants were $13.70 \pm 1.33(\mathrm{SD})$ for yoga group ranging from 14-17 years of age. Mean age for non-yoga group members were $13.60 \pm 0.97$ (SD) ranging from 12-17 years. Sixty percent were male and forty percent were female in both groups.

The mean $( \pm \mathrm{SD})$ height was slightly higher among the non-yoga group. There was no remarkable difference in mean weight of our participants among the two groups. BMI (Body Mass Index) of all participants were within the normal range. The BMI level was slightly higher among yoga group participants. From the table below it is clearly visible that in all aspects of selected anthropometric variables, there was no big gap among yoga and non-yoga group. The means and standard deviation of the anthropometric variables of the two group of are given below (Table 1).

Flexibility test score difference were observed among yoga group compared to non-yoga group. In yoga group, according to the test performed before and after yoga training, significant difference $(\mathrm{P}=0.017)$ were found in the score and the mean difference was -2.00 . This implies that an average of 2 inches of flexibility has increased among yoga group participants after the training. On the other hand, no significant difference in the mean score of flexibility test were observed among the non-yoga group participants (Table 2).

Improvement in balance were observed in the yoga group over 6 weeks period of yoga training session. Performance in the ST test had a mean increase from 24.10 seconds (Standard deviation [SD] $=16.33$ ) to 26.30 seconds (Standard deviation $[\mathrm{SD}]=17.33$ ) which was statistically significant $(\mathrm{P}=0.004)$. But no significant difference were found among the non-yoga group who performed the same test having regular forms of training (Table 3).

In addition to this, between group (yoga and no-yoga) comparison revealed that there was also significant difference in the SR test for flexibility $(\mathrm{P}=0.018)$ and $\mathrm{ST}$ test for balance $(\mathrm{P}=0.021)$ at the finishing of 6 weeks of yoga training period (Tables 2 and 3 ).

Table 1. Descriptive statistics of selected anthropometric variables.

\begin{tabular}{llclc}
\hline Variables & \multicolumn{2}{c}{ Yoga $(\mathbf{n}=\mathbf{1 0})$} & \multicolumn{2}{c}{ Non-yoga $(\mathbf{n}=10)$} \\
\cline { 2 - 5 } & Mean & SD & Mean & SD \\
\hline Height $(\mathrm{Cm})$ & 153.16 & 24.50 & 163.79 & 6.76 \\
Weight $(\mathrm{Kg})$ & 51.80 & 12.96 & 53.30 & 6.05 \\
BMI (Body Mass Index) & 22.23 & 3.61 & 19.88 & 1.98 \\
\hline
\end{tabular}

$\mathrm{SD}=$ Standard deviation, $\mathrm{n}=$ number of participants

Table 2. Analytical statistics for yoga and non-yoga traditional measures for flexibility.

\begin{tabular}{llll}
\hline \multirow{2}{*}{ Measures } & & \multicolumn{2}{c}{ Sit reach (SR) test (Inches) } \\
& Yoga Group & Non-yoga Group & Yoga - non-yoga Group \\
\hline Mean \pm SD (before) & $35.50 \pm 5.33$ & $37.20 \pm 5.03$ & $36.85 \pm 5.05$ \\
Mean \pm SD (after) & $38.50 \pm 6.62$ & $37.30 \pm 4.95$ & $37.90 \pm 5.72$ \\
Mean difference & -2.00 & -0.10 & -1.05 \\
Standard error of mean & 0.68 & 0.18 & 1.82 \\
t statistics & -2.93 & -0.56 & -2.58 \\
P value & $\mathbf{0 . 0 1 7}^{*}$ & 0.591 & $\mathbf{0 . 0 1 8}^{*}$ \\
\hline
\end{tabular}

\footnotetext{
* Significant level $\mathrm{p}<0.05$
} 
Table 3. Analytical statistics for yoga and non-yoga traditional measures for balance.

\begin{tabular}{llll}
\hline \multirow{2}{*}{ Measures } & \multicolumn{2}{l}{ Stork stand (ST) test (Seconds) } \\
\cline { 2 - 4 } & Yoga Group & Non-yoga Group & Yoga - non-yoga Group \\
\hline Mean \pm SD (before) & $24.10 \pm 16.33$ & $16.50 \pm 4.71$ & $20.30 \pm 12.33$ \\
Mean \pm SD (after) & $26.30 \pm 17.73$ & $16.30 \pm 4.52$ & $21.30 \pm 13.60$ \\
Mean difference & -2.20 & 0.20 & -1.0 \\
Standard error of mean & 0.57 & 0.13 & 0.40 \\
t statistics & -3.84 & 1.50 & -2.52 \\
P value & $\mathbf{0 . 0 0 4}$ & 0.168 & $\mathbf{0 . 0 2 1}^{*}$ \\
\hline
\end{tabular}

* Significant level $\mathrm{p}<0.05, \mathrm{SD}=$ Standard Deviation

\section{Discussion}

Flexibility and balance are important components of fitness of any sportsperson which plays an important role on their performance. In this particular study, our main focus was to determine if yoga has any additional effect on the flexibility and balance of shooting game athletes despite of their regular forms of training. Athletes from both groups took part in their regular training programs. Regular training session included warm up exercise, strength and endurance training, sport specific skill training and participating in their sport regularly. Only yoga group was given additional yoga training. Evidence have shown that flexibility training and yoga increases the range of motion of joints (McHugh and Cosgrave, 2010; Amin and Goodman, 2014) compared to those who are untrained. Therefore, we hypothesized that both group will show improvement in the flexibility test because all of them took part in regular warm-up sessions.

In our findings, it shows that in addition to the regular training session, yoga training significantly increases the measures of flexibility. On the contrary, the non-yoga group did not show any improvement in flexibility measures. Moreover, participants of yoga group also had higher flexibility than the participants of non-yoga group. Thus, 6 weeks of practicing yoga did help to improve flexibility measures in the actively training athletes while warm up stretching did not.

We have also hypothesized that both groups (yoga and non-yoga) will improve in balance test because participating regularly in strength and endurance training, sport specific skills particularly for shooting game provokes stability and balance (Zech et al., 2010). Furthermore, regularyoga practice supposed to increase the balance (Zech et al., 2010; Boehde et al., 2005), we expected that the additional yoga training will also improve the balance. Improvement in balance measures were observed significantly among yoga group according to our expectation. However, the non-yoga group failed to show any improvement. The effects of yoga was again revealed with significantly higher balance for yoga group versus non-yoga group. According to the findings above, we can again establish the fact that additional yoga training has positive effect on balance with traditional training even within shorter period of time (6 weeks).

Our study finds similarity with other studies too. In a study conducted among college athletes for about 10 weeks of yoga session in similar setting. Significant improvement were seen in both flexibility and balance among those participant who were belong to yoga group, in contrast, non-yoga group did not show any changes in their performance after the tests performed for measurement (Polsgrove et al., 2015). This evidence also supports our finding. Yoga can indeed enhance the flexibility and balance of athletes alongside with their traditional training.

Another study conducted among Olympic weightlifters revealed that 7-weeks of yoga training did not show any significant differences between groups on flexibility measures (Ernst and Jensen, 2016). Though the result contradicts the finding of our result, many other studies have shown the positive impact of yoga on flexibility. Hence, excluding only one evidence, we can surely recommend to include yoga session along with their regular training among athletes.

The athletes who practiced yoga for 6 weeks have shown the improvement in both flexibility and balance measures. According to our findings, we could expect that yoga group athletes would frequently exceed the nonyoga group athletes in flexibility and balance. Participating in additional training that focused on multiple components of fitness, may explain the improved measures of flexibility and balance for the yoga group athletes.

Based on the above cited literatures and consultations with many others the investigator confidently arrived at the conclusion that the trainee athletes who took part in the yoga session had enhanced flexibility and balance than the other group who took part only in the regular training, therefore, it would be beneficial if professional yoga expert can be included with the coaching team to provide regular training to improve the overall 
performance of shooting players. Furthermore, our study opened a room for further research in near future overcoming the limitation (time and samples size) revealed in this project. Our study population were mostly young adolescent. If we could include professional and adult sport persons, our result would have been different. Further research can also be done in other departments of BKSP adding more weeks of yoga training session with more outcome variables and measurement techniques to build the strong evidence. Yoga also has pshychological benefits which has not been studied in our study but this is also an important apsects to be added in future research.

\section{Conclusions}

Based on our findings, we can conclude that taking part in yoga session alongside with the traditional training methods has helped to improve flexibility and balance among shooting trainee athletes. Thus, yoga may support to enhance performance of those athletes by increasing specific components of fitness. Further studies to evaluate the impact of yoga including large sample size with different age groups, others port departments, other components of fitness and measurement tools, psychological aspects and more time may help to create more strong evidence. Hence, at this moment we can recommend that yoga professional could be included with the team to conduct yoga session on a regular basis to enhance performance of athletes. Coaches could also be trained on yoga poses so that they can conduct yoga session with their trainee athletes along with the other regular training.

\section{Acknowledgements}

We are grateful to the coaches and authorities at the shooting department of BKSP for their cooperation throughout the study period. We are also thankful to our participants for their active participation in this study that helped us to successfully complete the project.

\section{Conflict of interest}

None to declare.

\section{References}

Aaberg E, 2002. Strength, Speed and Power. Indianapolis (IN): Alpha.

Akhtar P, S Yardi and M Akhtar, 2013. Effects of yoga on functional capacity and well being. International Journal of Yoga, 6: 76.

Amin DJ and M Goodman, 2014. The effects of selected asanas in Iyengar yoga on flexibility: Pilot study. Journal of Bodywork and Movement Therapies, 18: 399-404.

Baechle T, 2008. Essentials of Strength Training and Conditioning. 3rd ed. Champaign (IL): Human Kinetics.

Bera TK and MV Rajapurkar, 1993. Body composition, cardiovascular endurance and anaerobic power of yogic practitioner. Indian Journal of Physiology and Pharmacology, 37: 225-225.

Boehde DD, JP Porcari, J Greany, B Udermann, D Johanson and C Foster, 2005. The physiological effects of 8 weeks of yoga training. Journal of Cardiopulmonary Rehabilitation and Prevention, 25: 290.

Broad WJ, 2012. The science of yoga: The risks and the rewards. Simon and Schuster.

Bryant CX and DJ Green, 2006. ACE personal trainer manual: The ultimate resource for fitness professionals. Recording for the Blind and Dyslexic.

Carrico M, 1997. Yoga journal's yoga basics: the essential beginner's guide to yoga for a lifetime of health and fitness. Macmillan.

Clark B and S Powers, 2012. The Complete Guide to Yin Yoga: The Philosophy and Practice of Yin Yoga. Ashland (OR): White Cloud Press.

Coulson M and D Archer, 2011. Practical fitness testing: Analysis in exercise and sport. Bloomsbury Publishing

Coulter H, 2010. A Manual for Students Teachers and Practitioners. Honesdale (PA): Body and Breath.

Ernst AT and RL Jensen, 2016. 7-weeks of yoga training and its effects on flexibility and rate of force development in olympic weightlifters. In ISBS-Conference Proceedings Archive, 34: 1.

Gulati R and A Sharma, 2011. Physical and mental fitness in sports person-role of yoga-fitness prescribe in ancient books. OvidiusUniv Ann Ser Phys Educ Sport SciMov Health, 1: 120-5.

Harrelson GL and E Swann, 2003. Yoga, Part I: An Introduction. Athletic Therapy Today, 8: 32-34.

Kaminoff L, A Matthews and S Ellis, 2007. Yoga anatomy. Champaign, IL: Human Kinetics.

McDermott KA, MR Rao, R Nagarathna, EJ Murphy, A Burke, RH Nagendra and FN Hecht, 2014. A yoga intervention for type 2 diabetes risk reduction: a pilot randomized controlled trial. BMC Complementary and Alternative Medicine, 14: 212. 
McHugh MP and CH Cosgrave, 2010. To stretch or not to stretch: the role of stretching in injury prevention and performance. Scandinavian Journal of Medicine and Science in Sports, 20: 169-181.

Pal A, N Srivastava, S Tiwari, NS Verma, VS Narain, GG Agrawal and K Kumar, 2011. Effect of yogic practices on lipid profile and body fat composition in patients of coronary artery disease. Complementary Therapies in Medicine, 19: 122-127.

Parikh HN, HM Patel, NR Pathak and S Chandwani, 2014. Effect of yoga practices on respiratory parameters in healthy young adults.

Polsgrove MJ, BM Eggleston and RJ Lockyer 2016. Impact of 10-weeks of yoga practice on flexibility and balance of college athletes. International Journal of Yoga, 9: 27.

Ross A and S Thomas, 2010. The health benefits of yoga and exercise: a review of comparison studies. The Journal of Alternative and Complementary Medicine, 16: 3-12.

Woodyard C, 2011. Exploring the therapeutic effects of yoga and its ability to increase quality of life. International Journal of Yoga, 4: 49.

Wolff M, K Sundquist, SL Lönn and P Midlöv, 2013. Impact of yoga on blood pressure and quality of life in patients with hypertension-a controlled trial in primary care, matched for systolic blood pressure. BMC Cardiovascular Disorders, 13: 111.

Zech A, M Hübscher, L Vogt, W Banzer, F Hänsel and K Pfeifer, 2010. Balance training for neuromuscular control and performance enhancement: a systematic review. Journal of Athletic Training, 45: 392-403. 